\title{
Edouard Glissant oder eine Reiseliteratur ohne Reisenden
}

Die Reiseliteratur ist im 21. Jahrhundert stärker als je zuvor. Sie kann als ein Seismograph von Entwicklungen gelten, welche die verschiedenen Regionen, Nationen und Areas, aber auch die Globalgesellschaft generell betreffen. Ein Dreivierteljahrhundert nach der Proklamation des Endes der Reisen durch Claude Lévi-Strauss in seinen Tristes Tropiques geht es dem Reisebericht besser denn je. Er repräsentiert jene literarische Form, in welcher wir viel über andere Kulturen und ferne oder nahe Länder, nicht zuletzt aber auch über uns selbst und die Grundlagen unseres Denkens erfahren. Reiseberichte sind aus der aktuellen Entwicklung der Literaturen der Welt nicht mehr wegzudenken.

Die seismographische Funktion von Reiseliteratur betrifft nicht zuletzt auch die Normen und Formen des Reiseberichts, die in einem weiten Maße zur Disposition gestellt worden sind und mit denen auf der Ebene der Formensprache äußerst spielerisch umgegangen wird. Beschäftigen wir uns also am vorläufigen Abschluss dieses letzten Teiles unserer Vorlesung mit einem Text, der jenes Element zur Disposition stellt, das fortzulassen in den bisherigen Reiseberichten eigentlich undenkbar war. Denn La terre magnétique von Edouard Glissant kommt ohne die Figur des Reisenden aus, ja streicht diese Figur explizit durch, um ein weiteres Mal in der Beziehung zwischen Reisen und Schreiben den Gegensatz zwischen Reisenden und Daheimgebliebenen zu akzentuieren. Dies ist eine ungeheure, unerhörte Neuerung, und Edouard Glissant führt sie in seinen Text, wie wir sehen werden, gleichsam 'natürlich' ein. Der frankophone karibische ${ }^{1}$ Dichter, Romancier und Theoretiker bietet uns eine Reiseliteratur, die - wie von ihm auch nicht anders zu erwarten - mit allen Wassern der Kultur- und Literaturtheorie gewaschen ist.

Der im Januar 2011 verstorbene martinikanische Schriftsteller, der aufgrund seines recht frühen, überraschenden Todes nicht mehr in den Genuss des Literaturnobelpreises kam, setzt in diesem Werk, das so etwas wie das literarisch-theoretische Testament des Autors darstellt, ganz auf die Kraft poetischer Erkenntnis. Er befindet sich damit im Einklang mit einer ganzen Vielzahl karibischer Autorinnen und Autoren, von denen hier nur José Martí, Saint-John Perse,

\footnotetext{
1 Zur Geschichte und den kulturellen Transferprozessen dieses Raumes vgl. Müller, Gesine: Die koloniale Karibik. Transferprozesse in hispanophonen und frankophonen Literaturen. Berlin: Walter de Gruyter 2012.

¿ Open Access. () 2020 Ottmar Ette, publiziert von De Gruyter. (cc) BY-NC-ND Dieses Werk ist lizenziert unter der Creative Commons Attribution-NonCommericial-NoDerivatives 4.0 Lizenz.

https://doi.org/10.1515/9783110650686-031
} 


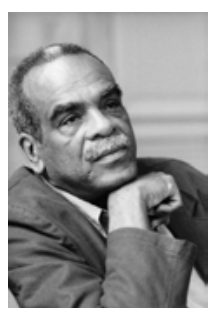

Abb. 95: Edouard Glissant (Sainte-Marie auf Martinique, 1928 - Paris, 2011).

Aimé Césaire, Maryse Condé oder vor allem auch José Lezama Lima genannt seien. Edouard Glissant ${ }^{2}$ konnte in der vierten Phase beschleunigter Globalisierung nicht nur auf den Theoremen und Denkstilen des französischen Poststrukturalismus und auf den Debatten rund um Aimé Césaires Négritude aufbauen, sondern vermochte auch, eine überaus kontinuierliche Traditionslinie für sein Schaffen fruchtbar zu machen, die bis hin zu den karibischen Kulturtheoretikern der Gegenwart führt, deren geistiger Vater er war. In Glissants reichem Schaffen kulminiert am Übergang vom 20. zum 21. Jahrhundert ein Denken weltweiter Komplexität, das in vielerlei Hinsicht nicht nur zukunftsträchtig ist, sondern die Zukunft zu bewahren hilft. Edouard Glissants theoretische Entwürfe eröffnen Spielräume, die das gegenwärtige Jahrhundert noch lange beschäftigen werden.

In seinem erstmals im November 2007 veröffentlichten poetischen Prosatext La terre magnétique. Les errances de Rapa Nui, l'île de Pâques (Das magnetische Land. Die Irrfahrten der Osterinsel Rapa Nui) erschien im Rahmen der vom Dichter selbst initiierten und realisierten Reisen unter dem Titel 'Les Peuples de l'Eau', die auch zu einer gemeinsamen Buchreihe führte und eine Vielzahl von Autoren weltumspannend mit der Welt der Inseln bekannt machte. ${ }^{3}$ In diesem letzten zu Lebzeiten des martinikanischen Essayisten und Philosophen veröffentlichten Band entwarf Glissant das poetische Bild einer Insel, die sich auf verschiedensten Ebenen - wie es schon der Untertitel dieses Werkes ankündigte in unsteter Bewegung befindet. Wieder stoßen wir hier, nunmehr zu Beginn des 21. Jahrhunderts, auf die Bewegungsfigur diskontinuierlicher, scheinbar zielloser

$2 \mathrm{Zu}$ dem martinikanischen Schriftsteller vgl. neuerdings das Edouard Glissant gewidmete Kapitel in Messling, Markus: Universalität nach dem Universalismus. Über frankophone Literaturen der Gegenwart. Berlin: Matthes \& Seitz 2019.

3 Vgl. hierzu meine ausführlichen Hinweise in verschiedenen Kapiteln zur vierten Phase beschleunigter Globalisierung in Ette, Ottmar: TransArea. Ich komme nochmals auf dieses Vorhaben zurück. 
Bewegungen, die sich bereits im Lexem Errances andeutet und die vom gesamten Text ausgeführt werden.

Diese 'Irrfahrten' der Osterinsel inmitten der sie umgebenden Meeresflächen weit draußen im Pazifik evozieren - für einen Dichter wie Glissant nicht ungewöhnlich - Arthur Rimbauds berühmtes Gedicht 'Le bateau ivre' und damit ein poetisches und poetologisches Befreiungspotenzial, welches der Band in seinem Fortgang nutzen wird. Diese Errances stehen dabei stets im Zeichen des Weltweiten, eines den gesamten Planeten umfassenden Koordinatensystems, innerhalb dessen die Insel zum im mehrfachen Sinne verrückten Fokus, ja zum sichtbaren Bezugspunkt des gesamten Erdballs, eben der terre in ihrer Globalität, wird. So heißt es in einer verdichteten, symbolisch aufgeladenen Passage dieses Textes:

Les oiseaux migrateurs apportent ici l'oeuf, dont le premier capté (qui contient le monde), après qu'on a dominé les courants de mer et le vertige de l'air, garantit le pouvoir pour l'année en cours. De même, la pierre ronde sacrée dite le nombril du monde prend la forme approximative d'un œuf, elle est polie et faite d'une matière qu'on ne trouve pas ailleurs dans l'île, et elle se trouve au bord de la mer et non pas au centre de la terre. Elle est au confluent des vents et des courants. ${ }^{4}$

Der Verweis auf die Zugvögel, die in den karibischen Literaturen eine lange Literaturgeschichte besitzen und etwa bei Fernando Ortiz in dessen Theorem der Transkulturalität eine entscheidende Rolle spielen, unterstreicht all jene Bewegungen und Mobilitäten, welche den gesamten Text durchziehen und auch etwa in den Meeresströmungen ihren Ausdruck finden. Sie verbinden die einsame Osterinsel mit den entferntesten Gegenden unseres Planeten. Doch gerade auch in den Strömungen der Lüfte sind die periodischen Migrationen der Zugvögel das, was biorhythmisch unsere Erde zusammenhält. Alle realen wie auch poetischen Verbindungen der Insel mit anderen Gestaden sind von Leben erfüllt.

Edouard Glissant gilt als der Dichter einer Poétique de la Relation, eines relationalen, archipelischen Denkens, das fernab von jeglichem Zentrum Beziehungsnetze zwischen den Inseln knüpft. Daher überrascht diese Passage zugleich, scheint sie sich doch einem Denken wieder zu öffnen, das doch von Glissant wortreich verabschiedet worden war. Denn besitzt die Welt also doch ein verborgenes Zentrum? Gibt es sozusagen einen 'Nabel der Welt', an dem sich alles ausrichtet?

4 Glissant, Edouard: La terre magnétique. Les errances de Rapa Nui, l’île de Pâques. En collaboration avec Sylvie Séma. Paris: Seuil 2007, S. 39. Übersetzung des Verf. 
Man würde den Kulturtheoretiker der Poétique de la Relation ${ }^{5}$ gründlich missverstehen, wollte man in dieser Passage die Abkehr von einem Denken vermuten, das sich über lange Jahrzehnte vehement gegen Strukturen zur Wehr setzte, die alles und alle zu zentrieren suchten. Nichts davon führt Glissant gleichsam durch die pazifische Hintertür der Osterinsel wieder ein. Denn dieser „Nabel der Welt“, von dem wir gleich eingangs erfahren, dass ihn von weither über den Pazifik gekommene japanische Pilger aufsuchen und verehren, ${ }^{6}$ bildet für Glissant sehr wohl einen Kreuzungspunkt aller Konfluenzen von Wasser, Luft und Erde, bündelt ein planetarisches Beziehungsgeflecht der vier Elemente, das zwischen den Luft- und den Meeresströmungen am Rande des magnetischen Landes der Osterinsel in einer dezentrierten Position entstand und mit einem alten Mythos verwoben wird, demzufolge die Zugvögel das Ei, das die Welt enthält, hierher, auf dieses Eiland, gebracht hätten.

Der gesamte Band des martinikanischen Kulturtheoretikers entfesselt eine wahre Poetik der Bewegung, in welcher die vernetzten Gewebe, Strömungen und Winde ständig en mouvement sind. Rapa Nui, die Osterinsel, wird von all jenen Bewegungen erzeugt, welche dieses Ei-Land kreuzen und durchqueren. Am Anfang, so erläutert uns dieser Ursprungsmythos, war die Bewegung: die Bewegungen der Zugvögel, die Bewegungen der Winde, die Bewegungen des Meeres. Und es entstand ein Land, eine Erde, die in und durch diese Bewegungen geschaffen wurde und lebte. Ein Land aus der Bewegung.

Halten wir also fest: Rapa Nui bildet kein übergeordnetes Zentrum, demgegenüber alles andere bloße Peripherie wäre. Aber man kann von Rapa Nui aus die Welt betrachten und aus dieser Perspektive neue Zuordnungen herstellen und ablesen. Unser Planet ordnet sich neu, sehen wir ihn erst einmal aus dem Blickwinkel der Osterinsel und nicht mehr, wie Glissant es zuvor stets getan hatte, aus der Perspektive der Karibik mit ihren archipelischen und transarchipelischen Vernetzungen. Die Osterinsel liegt weit draußen im Meer. Sie ist die isolierteste Insel unseres Erdkörpers.

Zugleich aber lässt der lyrische und vielfach fragmentierte Text Edouard Glissants von Beginn an keinen Zweifel aufkommen: Dieses Land ist mit der ganzen Welt, mit dem gesamten Erdball auf intimste Weise verbunden und verwoben. Die Osterinsel ist ein Mittelpunkt - aber in Form eines Schnittpunktes ohne Hierarchie, ohne Peripherie, ohne zentrierende Hysterie. Sie ist der Fluchtpunkt unserer Blicke, ganz so, wie es Jean Baudrillard in seinem Amérique mit dem Vanishing Point inmitten einer Landschaft tat, die wie die Wüste,

5 Vgl. Glissant, Edouard: Poétique de la Relation. Paris: Gallimard 1990.

6 Glissant, Edouard: La terre magnétique, S. 17. 
wie das Meer für den Menschen feindliche Anökumene ist. Doch sie kann gequert, sie kann von menschlichen Wegen durchlaufen werden.

Doch wer war zuerst: die Zugvögel oder das Ei? Die mehrfach beschworene Eiform - in der auch das berühmte Ei des Columbus und damit die planetarische Rundung der Welt wie die Macht über den Erdball mitbedacht sein mag und noch ein letztes Mal aufscheint - vereinigt die Bildung des Steines mit der alle Totalität umspannenden Form des Organischen und des Lebens schlechthin, erfahren wir doch an anderer Stelle, dass dieses Ei-Land einen (und folglich keineswegs den) Nabel der Welt enthält, un des nombrils du monde: Und diese sind „Orte des Todes und der Geburt“. ${ }^{7}$ Geburt, Sterben und Tod: Alles hängt miteinander zusammen und wird durch das Weiterleben untereinander verbunden. Das Ei steht wie das Eiland für die Welt, für das in ihm, für das in ihr Entstehende, birgt mit dem Leben aber zugleich immer auch den Tod, der in allem Leben ist und ihm niemals äußerlich bleibt. Im poetisch wie poetologisch verdichteten Text entsteht ein Lebenswissen und zugleich ein Weiterlebenswissen, das es beim Tode nicht belässt und Leben dort entstehen lässt, wo zuvor alles nur ein Vergehen war.

Das magnetische Land ist kein sicheres, gefestigtes Land: Seine Existenz bleibt stets prekär und gefährdet. Denn die Insel ist in ständiger Bewegung. Kann sie nicht jederzeit vom Meer verschlungen werden? Nicht umsonst wird die Vorstellung eingeblendet, dass die ganze Insel auf einem Süßwasserspiegel entlanggleite und dabei dem Verlauf der tektonischen Erdplatten folge: Die Insel ist folglich „ein umherirrendes Schiff, dessen Kurs allein die Zugvögel kennen“. ${ }^{8}$ Wie die Insel des heiligen Brandanus ${ }^{9}$ ist Rapa Nui immer in Bewegung, immer auf dem Weg. Sie ist eine der vielen beweglichen Inseln, die in der weltweiten Kartographie stets die Blicke der Entdecker auf sich gezogen haben und die Gemüter derer, die nach festen Ländergrenzen, nach festen Umrissen suchten, zutiefst beunruhigten.

Die solitäre Insel evoziert sofort das Bild des Schiffes, mit dem sie in den unterschiedlichsten Formen - sei es in der langen Tradition der schwimmenden Insel, sei es in der Kette von Transferprozessen, die jede Insel oder Inselgruppe erst konfigurieren - verbunden ist. Auf die Nähe von Glissants poetischem Text zu Anspielungen auf Arthur Rimbauds 'Le bateau ivre' wurde bereits aufmerksam gemacht. In der Doppelprojektion von Insel und Schiff, von der Insel als Schiff, wird dem Insularen jegliche Statik genommen: Die Insel ist nicht fest als Fels an

7 Ebda., S. 71.

8 Ebda., S. 41.

9 Vgl. Vázquez de Parga y Chueca, María José: San Brandán, navegación y visión. Aranjuez: Ediciones Doce Calles 2006. 
geologische Tiefen gefesselt, sondern navigiert und verliert sich im mobilen, von Strömungen dynamisierten Element des Meeres. Kein in der Tiefe verankerter Fixpunkt, sondern ein bewegliches Phänomen der Fläche, genauer: der Oberfläche. Das Schiff erscheint dergestalt als eine Bewegung an der Meeresoberfläche, die vom Schiff durchfurcht wird, das aber nicht in die Tiefe des Meeres eindringt. An dieser Stelle ergeben sich erneut philosophische Bezüge zu Baudrillards Band über die USA, ließe sich die Meereslandschaft doch ähnlich wie die Landschaft der Wüste als eine Landschaft der Theorie ansprechen, in welcher eine relationale Struktur zum Ausdruck kommt und sich die unterschiedlichsten diskontinuierlichen Bewegungen einschreiben und wieder verschwinden.

Die nur den Zugvögeln, nicht den Menschen bekannten Wege der Insel als Schiff auf einer Irrfahrt bewirken, dass die Insel zugleich von Dauer und vergänglich, dauerhaft und flüchtig ist: „Die Insel ist ephemer und verloren.“10 Nichts garantiert den Bestand der Insel und ihren Fortbestand. In diese flüchtige Beständigkeit, die gewiss auch jene der Literatur und des Schreibens selbst ist, schreiben sich die plattentektonisch getriebenen Bewegungen der Insel wie der Vorstellungen und Phantasien ihrer Bewohner ein:

L'île se déplace, de combien de centimètres par an, nul ne le sait, alors peut-être connaîtra-t-elle la destinée des terres archipéliques, engouffrées, un jour que nul ne sait non plus, dans les frottements inévitables des plaques des fonds, et l'immaginaire des Pascuans navigue dans l'espace du Pacifique et sous la lune du grand triangle, en quête de la parole perdue. C'est presque vrai ${ }^{11}$

Die Osterinsel ist ein Solitär, und doch teilt sie das Schicksal mit den Archipelen, in ihrer Relationalität und ihrer Ruhelosigkeit stets davon bedroht zu sein, unvermittelt in die Tiefe gerissen $\mathrm{zu}$ werden. Sie ist zweifellos eine Welt für sich, eine Insel-Welt, bildet aber mit all ihren Verbindungen durch Strömungen in Wasser und Wind eine Inselwelt mit all jenen Inseln zusammen, die weltweit über den Globus verteilt sind. Sie ist daher immer schon als Inselwelt Teil eines Transarchipels, das durch die Vielgestaltigkeit seiner Verbindungen und weltumspannenden Relationen bestimmt ist.

Doch die Insel steht auch in einer Beziehung zur Literatur, ja verkörpert poetische Verdichtung und eine Verbindung zum Mimetischen, die nicht auf reine Wahrheit zielt. Dieses Fast-Wahre, dieses presque vrai der Literatur, nimmt die Bewegungen der Insel und ihrer Bewohner auf und gibt beiden jenes 'verlorene Sprechen' wieder, wann und wo auch immer die Insel für immer im Meer versinken mag. Doch sie schwimmt noch immer, gleitet auf

10 Glissant, Edouard: La terre magnétique, S. 42.

11 Ebda., S. $48 \mathrm{f}$. 
den tektonischen Platten und bildet eine äußere Form heraus, die uns auf jeder Landkarte besticht. Denn ihre (zweifellos mit dem Attribut des göttlichen Auges versehene) Dreiecksform nimmt die Dreiecksform des gesamten polynesischen Archipels in sich auf und bildet somit das fraktale Muster einer Insel, die eine Insel der Inseln ist:

Triangle ouvert, c'est le triangle polynésien, qui marque à l'un de ses angles cet autre triangle, le plus éloigné et le plus solitaire, qui tient fermée la boucle, qui soutient toute cette surface, et qui est la terre magnétique. ${ }^{12}$

Das polynesische Dreieck zeichnet sich ein auf die Oberfläche des Meeres, schreibt sich ein auf die Oberfläche unserer Karten. In dieser Dreiecksform, die in der christlichen Ikonographie das Göttliche in seiner Anwesenheit repräsentiert, aber auch das Dreieck im Zentrum eines menschlichen Körpers sein könnte, vergegenständlicht und objektiviert sich eine Landschaft der Theorie, die im Rahmen jener Tradition, die den karibischen Raum schon so früh prägte, ganz selbstverständlich eine Theorie im weltweiten Maßstab ist. Im Zentrum dieser Theorie steht das Vielverbundene, das Archipelische und Transarchipelische, das einen Ort, eine Insel aus ihrer Multirelationalität heraus begreift und definiert.

Und vergessen wir dabei nicht, dass die Landschaft für Edouard Glissant zugleich Natur und Kultur ist - und damit letztlich auch etwas Lebendiges; so heißt es in Le discours antillais: „(Unsere Landschaft ist ihr eigenes Monument: Die Spur, die sie bedeutet, ist darunter spürbar. Es ist ganz Geschichte.)“13 Natur und Kultur sind nicht voneinander trennbar, wird Natur doch immer von der Kultur her gesehen und inszeniert, während die Kultur - wie schon in ihrem Gründungsbereich, der Agrikultur - vom Boden, vom physischen Fundament, von der Natur auf keine Weise zu trennen ist.

Der Begriff der Landschaft ist für Edouard Glissants Kulturmodell seit seinem Discours antillais von prägender Bedeutung. Landschaftselemente sind bei dem martinikanischen Dichter-Gelehrten stets Elemente einer Theorie

12 Ebda., S. 48. Zur offenen Strukturierung des Fraktalen vgl. Ette, Ottmar: WeltFraktale.

13 Glissant, Edouard: Le discours antillais. Paris: Gallimard - Folio 1997, S. 32; Verstärkend hierzu sei ein Zitat Edouard Glissants aus einer Pressekonferenz am 26. Juni 2006 im MadisonHotel in Berlin genannt: „Dans la Caraïbe comme en général dans les Amériques le paysage est le véritable monument historique et cette dimension-là a beaucoup influencé ce que je fais en poésie. Le paysage devient un personnage à la fois des romans et de la pensée et de la poésie. C'est pourquoi dans tout ce que j'ai écrit, romans poésie, essais, le paysage est un personnage vivant.“ 
lebendigen, aus dem Leben selbst kommenden Schreibens. Texttheoretisch klingt dies bei ihm dann so:

Je ne sais pas à quel âge, dans mon très jeune temps, j’ai rêvé d'avoir développé un texte qui s'enroulait innocemment mais dans une drue manière de triomphe sur lui-même, jusqu'à engendrer au fur et à mesure ses propres sens. La répétition en était le fil, avec cette imperceptible déviance qui fait avancer. Dans ce que j'écris, toujours j'ai poursuivi ce texte. Je m'ennuie encore de ne pas retrouver l'enhalement tant tourbillonnant qu'il créait, qui semblait fouiller dans une brousse et dévaler des volcans. Mais j'en rapporte comme une ombre parfois, qui relie entre elles les quelques roches de mots que j'entasse au large d'un tel paysage, oui, une brousse, sommée d'un volcan. ${ }^{14}$

Dieses Zitat verdeutlicht die enorme Bedeutung der Landschaft für die Poetik und Poetologie von Edouard Glissant. Theorie verkörpert sich hier in ganz bestimmten Landschaftselementen, erscheint symbolhaft für bestimmte Theorieansätze, die der martinikanische Philosoph und Poet dann theoretisch und dichterisch durchbuchstabiert. Das (lebendige) Dreieck der Insel Rapa Nui im Dreieck des polynesischen Archipels ${ }^{15}$ bildet die fraktale Konfiguration nicht allein der Insellandschaften des Pazifik, sondern beinhaltet zugleich als Eiland in der Eiform des von Zugvögeln (hervor)gebrachten Eis jenen Nabel der Welt, von dem aus die Rundung der Erde gedacht und in ihren weltweiten Dimensionen überdacht werden kann. Die Osterinsel wird zum Land und zum Eiland, aber auch im Sinne des Lexems terre zur Erde und zum Erdball. Rapa Nui tritt an die Stelle der gesamten Erde, wird als pars pro toto Stellvertreterin des gesamten Planeten.

Wir hatten die grundlegende Ambivalenz der Inselmetaphorik bereits angedeutet und wollen hier noch deutlicher werden. Denn einerseits ist die Osterinsel auf eine geradezu extreme Weise eine Insel-Welt, die eine in sich abgeschlossene Welt mit ihrem eigenen Raum, ihrer eigenen Zeit, ihrem eigenen Klima und folglich auch ihren eigenen Bewegungsmustern repräsentiert. Wie keine andere Insel auf diesem Planeten ist sie - wie gleich zu Beginn des Bandes betont wird - von anderen Ufern, von anderen Ländern durch gewaltige Distanzen getrennt und damit in einem grundlegenden Sinne isoliert. ${ }^{16}$

14 Glissant, Edouard: La Cohée du Lamentin. Poétique V. Paris: Gallimard 2005, S. 20.

15 Vgl. zur spezifischen Problematik Rapa Nuis im Schnittpunkt unterschiedlicher Geschichten und Insel-Projektionen McCall, Grant: Rapanui: Traum und Alptraum. Betrachtungen zur Konstruktion von Inseln. In: Weinhäupl, Heide / Wolfsberger, Margit (Hg.): Trauminseln? Tourismus und Alltag in Urlaubsparadiesen. Wien: Lit Verlag 2006, S. 263-278.

16 Vgl. Glissant, Edouard: La terre magnétique, S. 10. 
Diese Isolation hat Folgen. Und diese Folgen reichen weit ins Experimentelle der Reiseliteratur hinein, weiß Glissant doch aus einem faktischen Nachteil, der Isoliertheit und schweren Erreichbarkeit der Insel, einen theoretischen Vorteil zu ziehen. Die isolierte Abgelegenheit der Insel ist ein Faktum, das in der Darstellung der Genese des Textes auch durch die Tatsache bewusst in Szene gesetzt wird, dass es dem Dichter in seinem fortgeschrittenen Alter nicht mehr möglich war, eine so weite und anstrengende Reise wie die zur Osterinsel selbst in Angriff zu nehmen. Er blieb folglich zuhause und gab seinen anvisierten Status als Reisender auf. Damit trennte er das Reisen vom Schreiben auf eine theoretisch zugleich sehr anspruchsvolle Art und Weise, schrieb er doch nun über die Osterinsel, ohne selbst zu ihr gereist zu sein. Wie war dies möglich? Und was ist ein Reisebericht, der von einem verfasst worden ist, der gar nicht zum Gegenstand der Reisebeschreibung reiste?

Die praktische Lösung war zunächst sehr einfach. So sollte anstelle Edouard Glissants dessen Lebenspartnerin Sylvie Séma die Reise unternehmen und den zuhause gebliebenen Verfasser dieses poetischen Reiseberichts durch Skizzen und Notizen, durch Zeugnisse und Zeichnungen mit jenen Grundlagen für ein Schreiben versorgen, das explizit auf die Beglaubigung durch das eigene InAugenschein-Nehmen verzichtet, um von einem anderen Ort des Schreibens aus diese Welt literarisch zusammenzufügen. Une écriture peut en cacher une autre.

La terre magnétique ist folglich ein Reisebericht, der nicht auf der Reise des Reiseschriftstellers aufruht, sondern von einem im wahrsten Sinne des Wortes Daheimgebliebenen stammt. Jene beiden Pole, die innerhalb der langen Geschichte abendländischer Reiseliteratur stets sorgsam auseinander gehalten wurden, fallen auf diese Weise in eins. Die Funktionen von Reisendem und Schreibendem werden weitgehend aufgetrennt und neu miteinander kombiniert. Was daraus entsteht, ist ein Reisebericht von einem, der eine andere aussandte, für ihn die Reise anzutreten. Edouard Glissant testet aus einer theoretisch aufgeklärten Sicht die Dehnbarkeit des Autorbegriffs. Und wirft dabei Normen der Mimesis, der Auctoritas und der Glaubhaftigkeit über Bord. Sein Reisebericht avanciert damit zu einem Experiment im Schatten der Frage, was denn ein Autor, was denn ein Reiseschriftsteller eigentlich sei.

Die Konsequenzen für die Gattung des Reiseberichts liegen auf der Hand. Denn damit werden deren Grundlagen insofern aufgekündigt, als der Schreibende auf den Bericht einer - ihm freilich sehr vertrauten - Reisenden wie auch auf andere Zeugnisse zurückgreift, die ihm zur Verfügung stehen oder gestellt werden. Das von Sylvie Séma, der auf die Osterinsel stellvertretend Reisenden, Vorgefundene wird mit dem am heimischen Schreibtisch Erfundenen zu etwas gemeinsam Hergestelltem und mehr noch gemeinsam Erlebten, das sich aus unmittelbaren wie mittelbaren Quellen speist. 
Dabei sei nicht verschwiegen, dass dem Text zugleich eine geradezu testamentarische Dimension zuwächst, insofern der Schriftsteller aus der Perspektive der Reisenden in eine 'andere Welt' rückt, als wollte er die Wege der Reisenden aus einem Jenseits kommentieren und mit seinem literarischen Wort - dem einst verlorenen Wort, auf dessen Suche sich die Insel gemacht hat - begleiten. Durch den Tod des Schriftstellers wenige Jahre später ist diese ganz eigene Dimension des Textes offenkundig und folglich lesbar geworden. La terre magnétique bildet das literarisch-poetologische Testament von Edouard Glissant aber auch in jenem Sinne, als sich in den Inselbildern der Osterinsel ebenso Glissants Discours antillais wie seine Poétique de la Relation in verdichteter Form und wie in einer mise en abyme finden. In der Insel der Inseln besucht der martinikanische Poet noch einmal, noch ein letztes Mal, jene Welt der Archipele, die er ausgehend von den Inseln seiner Antillen auf eine weltumspannende Folie gleichsam transtropisch projiziert hat. Auch in diesem Sinne ist sein Insel-Buch ein literarisches wie philosophisches Vermächtnis.

Doch versuchen wir, unseren Gedanken an die Insel-Welt noch in dem bereits angedeuteten Sinne zu vervollständigen. Denn andererseits ist diese geographisch extrem isolierte, eine eigene Welt für sich bildende Insel nicht nur eine abgeschlossene Insel-Welt, sondern zugleich eine Inselwelt, insofern sich in ihr eine ganze Welt von Inseln überlagert und bündelt. So schaffen sich in dem kleinen Eiland Rapa Nui mit seinen Vulkanen die vier Elemente von Feuer und Erde, Luft und Wasser in den Meeres- und Luftströmungen, aber auch in den Bewegungen der tektonischen Erdplatten wie des feurigen Magmas, das mit dem pazifischen Feuerring verbunden ist, einen Bewegungs-Ort vielfältigster planetarischer Konfluenzen, an dem sich eine Welt von Inseln immer wieder neu konfiguriert. Es ist eine Welt in absoluter Bewegtheit, erfüllt von den vier Elementen in Bewegung, welche diese Inselwelt als Kosmos - und dies bedeutet etymologisch als Ordnung und als Schönheit - entwerfen.

Doch mit der Kombination von Insel-Welt und Inselwelt ist es in diesem Insel-Buch über die Osterinsel noch nicht genug. Denn die mise en abymeStruktur des Textes und seiner Insel macht uns noch auf eine weitere Dimension aufmerksam. Rapa Nui wird in diesem Sinne als fraktale Vervielfachung des Insularen zu einer InselInsel, ${ }^{17}$ in der sich nicht nur die verschiedensten Inseln Polynesiens überkreuzen und überschneiden, sondern das vielgestaltige Gemachtsein dieser (Poly-) Insel aus anderen Inseln noch dadurch vervielfacht

17 Zum Begriff der InselInsel vgl. das siebte Kapitel in Ette, Ottmar: ZusammenLebensWissen. List, Last und Lust literarischer Konvivenz im globalen Maßstab (2010). 
wird, dass die von der Lebenspartnerin des Erzählers bereiste Insel vom Erzähler selbst von anderen Inseln aus - seien es die der Antillen oder der Ile de France - niedergeschrieben und weltweit verwoben wird. Die ganze Welt in einer Insel, die die ganze Welt ist, ohne doch deren Zentrum zu sein oder sein zu wollen. In La terre magnétique bündelt sich alles.

Doch kommen wir an dieser Stelle unserer Überlegungen noch einmal auf die so ungewöhnliche Auftrennung der Funktionen von Reisen und Schreiben sowie auf das neue Verhältnis zurück, das Reisen und Schreiben in diesem Text miteinander eingehen. Wir sollten nicht der Verlockung erliegen, der weiblichen Reisenden das Auffinden und Erleben der Osterinsel, dem männlichen Schriftsteller aber als Schöpfer und Demiurgen das Erfinden und Durchdringen des Gegenstands zuzuordnen. Eine derartige geschlechterstereotype Aufspaltung wäre dem Band unangemessen.

Denn bei beiden, ebenso der Figur der Reisenden wie der Figur des Daheimgebliebenen, bilden sich Finden, Erfinden und Erleben in einem intensiven Wechselspiel so heraus, dass es nicht der bis zum Ende des 18. Jahrhunderts vorherrschenden epistemologischen Trennung zwischen voyageur und philosophe entspricht, zwischen dem vermeintlich zufällig und planlos sammelnden Reisenden und dem im Zentrum des Wissens situierten Philosophen, der das so Gesammelte erst in ein System, in eine klare und durchdachte Ordnung überführt. War nicht die erste Karte der Inselwelt der Karibik im Jahre 1500 von der Hand des großen Steuermannes Juan de la Cosa so entworfen worden, dass in ihr die Inseln im Zentrum der neuen Welt gleichsam aus den mittelalterlichen Portulanen aufstiegen, um sich gemeinsam mit den alten Mythen Asiens auf einer Weltkarte wiederzufinden, in der sich das Aufgefundene und Erlebte mit dem Erfundenen ebenso präzise wie phantasiereich verband? Waren es nicht jene mobilen Inseln gewesen, mit deren Geschichte sich Alexander von Humboldt intensiv in seinem Examen critique beschäftigte, welche den Entdeckern mögliche Wege nach Westen wiesen, unabhängig davon, ob sie in den neu aufgefundenen Inseln den extremen Osten des Orients oder wenig später eine neue, den Europäern noch unbekannte Welt erahnten? Stets war das Vorgefundene vom eigentlich Erfundenen nicht fein säuberlich zu trennen gewesen und hatte als Konglomerat und Amalgam doch dem Erlebten als Bezugspunkt gedient.

Die komplexe Relationalität der Textgenese ist für Glissants La terre magnétique von großer Relevanz, so dass der erste lange Satz des incipit dieses Prosabandes einer so vielschichtigen semantischen wie lebensweltlichen Beziehung bewusst gewidmet wird. Der Text setzt folglich etwas schwerfällig, ja sperrig ein, wie es freilich für Schriften des martinikanischen Dichters recht charakteristisch ist: 


\begin{abstract}
Nous étions convenus de travailler en relais et de fréquenter l'île de ces deux manières qui peut-être se compléteraient : Sylvie sur ce qu'il fallait bien appeler le terrain (elle prendrait l'avion jusqu'à Santiago, elle voulait absolument pousser jusqu'à Valparaíso, vers ce rêve commun à toutes les enfances du monde, elle continuerait ensuite à l'île de Pâques, vingttrois heures de vol au moins), et moi par les commentaires que je ferais de ce qu'elle enverrait et de ce qu'elle rapporterait, notes, impressions, dessins, films et photos, et par l'ordre ou le désordre de littérature qu'avec son aide j'apporterais à ces documents et à son sentiment ainsi abruptement saisi. ${ }^{18}$
\end{abstract}

Reisen und Schreiben erscheinen in dieser Passage als komplementäre Praktiken. Die lange Flugreise der Gefährtin erscheint hier als eigentliche Traumreise, welche dem alten Dichterfürsten fortan verwehrt ist. Er muss zuhause bleiben. Doch die Arbeitsteilung der beiden wirft Fragen auf, verweist sie doch auf ein wissenschaftliches Modell, wie es das ausgehende 18. und beginnende 19. Jahrhundert entwickelte und wie wir es in dieser Vorlesung wiederholt studiert haben. Denn die Feldforschung obliegt Sylvie, während deren Auswertung Edouard übernimmt. Soweit, so gut. Allerdings erscheint die Transposition durch den Literaten als ein in die Ordnung oder Unordnung der Literatur Bringen, als eine Arbeit, die keinen wissenschaftlichen, sehr wohl aber poetischen Normen und Formen folgt. Zugleich wird der Autor nicht zum Auctor, sondern zum Commentator, der über eine Auctoritas sekundärer Ordnung verfügt.

Am Ende des dritten von insgesamt sechs Teilen dieses Bandes - und damit genau im Zentrum der gesamten Textstruktur von La terre magnétique lässt sich eine bemerkenswerte Konfluenz der verschiedenen Blicke und Blickrichtungen feststellen, die den Text in ihrer Vereinigung zeugen und erzeugen. Die Vielgestaltigkeit dieser Konfluenzen durchzieht den gesamten Band von Beginn an ebenso wie das fraktale Muster einer Insel, die in der lyrischen Prosa dieser verdoppelten Reise zur Osterinsel von Beginn an diese Insel mit der Welt verknüpft. Immer wieder weitet sich die Perspektivik, werden explizite Beziehungen der Osterinsel nicht allein zum polynesischen Archipel und nach Tahiti, sondern auch zum amerikanischen Mittelmeer der Antillen wie zum europäischen Mittelmeer mit seinen Inseln Sardinien und Korsika, aber auch nach Island hergestellt, das schon Columbus auf seinen frühen Fahrten kennengelernt hatte. ${ }^{19}$ Eine Welt der Inseln wird entworfen, welche keine Kontinente, kein Kontinuierliches benötigt. In einer solchen Welt ist das Erleben diskontinuierlich, ist die Kontinuität von Erfahren und Erleben ständig durchbrochen. Und mit ihr die Verstehensstrukturen des Bandes.

18 Glissant, Edouard: La terre magnétique, S. 9.

19 Ebda., S. $62 \mathrm{f}$. 
Was aber vermag ein Reisebericht zu sein, der auf eine eigentümliche Art und Weise exzentrisch ist? In welchem der Autor nicht reist und zum Kommentator einer anderen Reisenden wird, die ihm zwar Material, aber keine Autorschaft liefert? Das Ergebnis eines solchen Experiments ist eine Außerhalbbefindlichkeit mit Blick auf den Gegenstand, die Reise auf die Osterinsel, und das Schriftstellersubjekt eines Reisenden, den es nicht gibt, der sich zu keinem Zeitpunkt in Bewegung setzte. Aus dieser doppelten Exzentrizität, aus dieser doppelten Außerhalbbefindlichkeit generiert der Text seine Poetologie, die Grundlagen eines fraktalen Verstehensmodells, das in einer Insel alle Inseln umgreift.

Auf diese Weise generiert und manifestiert sich eine transarchipelische Sicht, die sich über alle „Routen der Welt“20 von Insel zu Insel, von Archipel zu Archipel so entfaltet, wie der französische Dreimaster La Boudeuse unter der Schirmherrschaft der UNESCO und unter dem Kommando von Kapitän Patrice Franceschi am 25. Juli 2004 vom korsischen Bastia aus eine Weltumsegelung in Angriff nahm, in deren Verlauf von 1063 Tagen und etwa 60000 zurückgelegten Kilometern zwölf verschiedene Schriftsteller und Journalisten, die von Edouard Glissant zuvor ausgewählt worden waren, jeweils Expeditionen zu einzelnen 'Völkern am Wasser' unternahmen, bevor das französische Segelschiff am 25. Juni 2007 - und folglich nur wenige Monate vor Erscheinen von La terre magnétique - nach Korsika zurückkehrte. Soviel zum materiellen Rahmen von Glissants Vorhaben 'Les Peuples de l'Eau', seines großen Insel-Projekts.

Dieses Insel-Projekt Edouard Glissants ist vom Besuch Rapa Nuis letztlich durch Sylvie Séma nicht zu trennen. Denn die treibende Kraft hinter allen Reisen und den dazwischengeschalteten Insel-Aufenthalten der Literaten war kein anderer als der martinikanische Dichter, der die Fäden für alle Reisen gleichsam in seinen Händen hielt. Doch das literarische und philosophische ist vom maritimen Vorhaben ebenso wenig isolierbar wie die Osterinsel von den anderen der auf dieser Seefahrt besuchten Inseln: Die Insel und das Schiff reflektieren sich wechselseitig und setzen die unterschiedlichsten Transferprozesse in Szene. Der in Zusammenarbeit mit Sylvie Séma entstandene Band Edouard Glissants bildet nicht nur von seinem Anspruch, sondern auch von dessen Einlösung her - eine offene Strukturierung, die als Band die verschiedenen Bände, die unterschiedlichen Reisen der beteiligten Autoren, auf wohlkalkulierte Weise miteinander verbindet. Dies also ist die Grundstruktur einer Reise im Kontext der vielen Reisen, als deren Drahtzieher Edouard Glissant gelten darf. Wer also ist der Autor einer Reise? Muss dieser Autor notwendig mit dem Reisenden zusammenfallen?

20 Ebda., S. 63. 
Die Antwort auf diese Frage, erst einmal vor diesem Hintergrund so gestellt, ist einfach: Nein, der Autor muss nicht der Reisende sein. Der Autor kann auch eine panoramatische Position besetzen, die zugleich auch eine metatextuelle ist. Die relationale und zugleich transarchipelische Sichtweise, die sich immer wieder gerade zwischen der Osterinsel und den Antillen entwickelt, prägt die poetische und poetologische Prosa Edouard Glissants und knüpft zweifellos an seine berühmte 'Poetik der Relation' an, die er ausgehend von den Antillen zunächst innerarchipelisch entwickelte, bevor er sie hemisphärisch auf den gesamten amerikanischen Kontinent ausweitete.

In seiner 1981 in Le discours antillais angelegten und 1990 in Poétique de la Relation entfalteten Theorie, die sich in einem kritischen Dialog mit wesentlich stärker zentrierenden Vorstellungen schärfte, wie sie Jean Bernabé, Patrick Chamoiseau und Raphaël Confiant in ihrem vielbeachteten, aber auch vielüberschätzten Eloge de la créolité von 1989 ausformulierten, ${ }^{21}$ ließ Glissant keinen Zweifel daran aufkommen, dass seine Raumkonzeption der Antillen zugleich relational und hemisphärisch gedacht war. Denn Glissant begriff die Antillen als „Multi-Relation“, die keineswegs als verstreute Fleckchen Erde in einem „See der USA“ zu begreifen seien, sondern gleichsam den „Ästuar der Amerikas““22 bildeten. Es ist, als hätte Edouard Glissant den anspruchsvollen Versuch unternommen, jene Landschaft der karibischen Dichter und Theoretiker von José Martí und Fernando Ortiz über Saint-John Perse und Aimé Césaire bis hin zu Derek Walcott und Maryse Condé zu einem literarischen Höhepunkt zu führen, an welchem diese Theorie zu einer weltumspannenden Kulturtheorie wird. Dieser Höhepunkt ist mit dem experimentellen Reisebericht La terre magnétique erreicht.

Die hemisphärische Sicht weitet sich in Das magnetische Land konsequent zu einer transarchipelischen Dynamik, deren Relationalität sich nunmehr weltweit entspannt und zugleich auch den amerikanischen Kontinent umfasst: ein Polynesien, ein Vielinselland im globalen Maßstab. Dies zeigt der bereits angesprochene Mikrotext im Zentralstück des gesamten Bandes mit seiner makrogeographischen Dimensionierung mit größtmöglicher Präzision. Dort heißt es in einer lyrischer Verdichtung, die etwas von den Versen des Kubaners José Martí, aber auch von Roland Barthes' Mikrotexten En Grèce hat:

21 Vgl. Bernabé, Jean / Chamoiseau, Patrick / Confiant, Raphaël: Eloge de la Créolité (1989); vgl. hierzu auch das elfte Kapitel meines bereits erwähnten Bandes Literatur in Bewegung (2001).

22 Glissant, Edouard: Le discours antillais, S. 249. 
Etre Rapa Nui, dépositaire de l'unique et du très commun, ces forces qui ont porté les peuples du Pacifique et de l'Amérique du Sud. [...] Papa Kiko chante un complaint des Quechuas du haut des Andes et il dans à peu près un pas tambouré de Vanuatu, avec une totale profondeur. Pirù perfectionne ses ramassages de déchets, malgré les débordements incessants. Le corps-île de l'île est en eux, dont les secrets ont résidé circulé dans les veines des volcans des habitants, inséparables. C'est parce qu'elle est si loin de toute mesure et de tout calcul et du toute vue et de toute approche, à jamais dans l'angle d'en haut, qui a favorisé de ses dons les archipels rassemblés là en bas. ${ }^{23}$

Eine Welt der Inseln und zu Inseln gewordener Kontinente beginnt sich hier zu runden. Alles ist mit allem verbunden, alle Stimmen überlagern sich zu einem vielstimmigen Chor, der sich über die Meere dieser Welt verbreitet: eine Polyphonie, die von Polynesien aus weltumspannend ertönt. Die Verbindung der aufgrund der gewaltigen Distanzen scheinbar isolierten Insel-Welt mit den Inselwelten der antillanischen Archipele, aber auch den Anden des kontinentalen Amerika lässt eine Welt entstehen, die im Blick von oben wie aus der Perspektive des Schöpfers die dynamische, mobile Relationalität eines Planeten hervorbringt, in der die Gesänge räumlich weit voneinander entfernter Kulturen von verschiedenen Punkten aus hörbar werden, ohne doch miteinander zu verschmelzen. Wie in Jorge Luis Borges' El Aleph ist alles sinnlich gleichzeitig wahrnehmbar, doch stürzen die Klänge (wie bei Borges die Bilder) keineswegs ineinander: Alles ist vielstimmig hör- und begreifbar.

Die offenkundig transkulturelle Anlage dieser polyphonen Orchestrierung von Pazifik und Amerika dynamisiert eine transareale Modellierung im weltweiten Maßstab. Von der Insel-Welt und Inselwelt der Osterinsel wird das Archipele und Kontinente miteinander verbindende Planetarische - und dies eröffnet eine geradezu österliche Dimension - neu begreifbar, neu erlebbar, neu lebbar. Wir wohnen einer Konvivenz der Stimmen, der Klänge bei.

Edouard Glissant ist zweifellos am Ende seines Lebens noch ein letzter, kulturtheoretisch klug vorbereiteter Coup gelungen, indem er seine antillanische Inselwelt in der transpazifischen Insel-Vielheit gleichsam transarealisierte. Die Konvivenz der Stimmen verwandelt die Polyphonie keineswegs in ein Unisono, in eine Ein-Tönigkeit, sondern macht gerade die Differenzen stark, die das Rückgrat der transarchipelischen Vielstimmigkeit bilden. Das Zusammentönen, das Zusammenklingen bildet aber auch eine gelingende Konvivenz, die sich in einem weltumspannenden Maßstab auf diesen Seiten abzeichnet. Genau hierin, so scheint mir, liegt das Vermächtnis dieses Reiseberichts, der ohne einen Reisenden auskommt.

23 Glissant, Edouard: La terre magnétique, S. 92. 
In jüngster Zeit haben sich wieder die Stimmen gemehrt, die die Reiseliteratur als Auslaufmodell sehen oder ihr einen baldigen Tod vorhersagen. ${ }^{24}$ Ich würde dies als ein gutes Zeichen werten. Denn hatte nicht Claude Lévi-Strauss in seinen Traurigen Tropen das baldige Ende der Reisen und der Reiseliteratur auf höchst kreative Weise, aber fälschlich prognostiziert? Wir haben im Verlauf unserer Vorlesung gesehen, das sich gleichzeitig und nachfolgend in Europa wie in Amerika eine Vielzahl von Reiseberichten entwickelte, die lebendiger als jemals zuvor die Normen wie die Formen des Reiseberichts in Frage stellten und mit höchst unterschiedlichen Formen experimentellen Reise-Schreibens die Vitalität der Gattung eindrucksvoll unter Beweis stellten.

Nach dem Ende der vierten Phase beschleunigter Globalisierung, das wir in den zurückliegenden Jahren miterleben durften, scheint auch für den Reisebericht des 21. Jahrhunderts eine neue Epoche angebrochen. Doch der Reisebericht erfüllt heute lebendiger denn je seine ihm historisch zugewachsene Funktion, ein Seismograph der gesamtgesellschaftlichen Entwicklungen und Veränderungen zu sein. Im Vorfinden und Erfinden dessen, was er uns vorlegt und unserem Erleben und Erlebenswissen anheimstellt, sehen wir prospektiv die Konturen jener Literaturen der Welt aufscheinen, die sich in Zukunft herausbilden werden. Das Vorgefundene wie das Erfundene friktional einem Erleben zuzuführen, das in der Vielfalt und Vielstimmigkeit der literarischen Formen seinen ästhetischen Ausdruck findet, ist im genießenden Verstehen des Reiseberichts aufgehoben. Im literarisch vermittelten Erleben und Nacherleben der Reisen, dies versuchte unsere Vorlesung zu zeigen, sind den Beziehungen zwischen Reisen und Schreiben keine unüberwindlichen Normen gesetzt. Wohl aber ist der Lust am Text im experimentellen Umgang mit unendlichen reiseliterarischen Formen der Weg bereitet. Machen wir uns an diesem Ausgang unserer Vorlesung also lustvoll auf den Weg zu neuen Horizonten!

24 Macfarlane, Robert: Is Travel Writing Dead? In: Granta (London) (2019) <http://granta. com/travel-writing-dead-robert-macfarlane> (Zugriff 1.4.2019). 